\title{
Comparison of Infection between Internal-External and External Percutaneous Transhepatic Biliary Drainage in Treating Patients with Malignant Obstructive Jaundice
}

\author{
Chuan Xu' ${ }^{1}$, Xin-En Huang ${ }^{2 *}$, Shu-Xiang Wang ${ }^{1 *}$, Peng-Hua Lv ${ }^{1}$, Ling Sun ${ }^{1}$, \\ Fu-An Wang ${ }^{1}$
}

\begin{abstract}
Purpose: Percutaneous transhepatic biliary drainage (PTBD) is a form of palliative care for patients with malignant obstructive jaundice. We here compared the infection incidence between internal-external and external drainage for patients with malignant obstructive jaundice. Methods: Patients with malignant obstructive jaundice without infection before surgery receiving internal-external or external drainage from January 2008 to July 2014 were recruited. According to percutaneous transhepatic cholangiography (PTC), if the guide wire could pass through the occlusion and enter the duodenum, we recommended internal-external drainage, and external drainage biliary drainage was set up if the occlusion was not crossed. All patients with infection after procedure received a cultivation of blood and a bile bacteriological test. Results: Among 110 patients with malignant obstructive jaundice, $22(52.4 \%)$ were diagnosed with infection after the procedure in the internal-external drainage group, whereas $19(27.9 \%)$ patients were so affected in the external drainage group, the difference being significant $(p<0.05)$. In 8 patients $(36.3 \%)$ in the internal-external group infection was controlled, as compared to $12(63.1 \%)$ in the external group $(p<0.05)$. The mortality rate for patients with infection not controlled in internal-external group in one month was $42.8 \%$, while this rate in external group was 28.6\% ( $p<0.05)$. Conclusion: External drainage is a good choice, which could significantly reduce the chance of biliary infection caused by bacteria, and decrease the mortality rate at one month and improve the long-term prognosis.
\end{abstract}

Keywords: Internal-external drainage - external drainage - infection incidence - malignant obstructive jaundice

Asian Pac J Cancer Prev, 16 (6), 2543-2546

\section{Introduction}

Percutaneous transhepatic biliary drainage (PTBD) is becoming more and more useful as a palliative treatment for patients with malignant obstructive jaundice, which could not only significantly reduce bilirubin levels, improve liver function and prolong survival time, but also be used as a preoperative drainage, provide the conditions for subsequent treatment (De et al., 2013). But several studies have confirmed that biliary infection after PTBD is a major independent risk factor affecting patients with malignant obstructive jaundice survival time, which could damage the patient's liver function, reduce survival quality, shorten the life time (Kawakami et al., 2011; Yu et al., 2010). In our center, if the guide wire could pass through the occlusion and into the duodenum, we prefer to use internal-external drainage, while the occlusion is not crossed, external drainage biliary catheter is placed. We think that internal-external drainage in the treatment of malignant obstructive jaundice not only leading bile flow into intestine, maintaining the physical channel of bile drainage, also declining bilirubin level quickly which is beneficial to the recovery of liver function and reduce the gastrointestinal complications caused by lack of bile (Xu et al., 2014 ; Xu et al., 2014 ; Xu et al., 2014). In order to compare the infection incidence between internal-external and external percutaneous transhepatic biliary drainage for patients with malignant obstructive jaundice, we collect patients with malignant obstructive jaundice receiving these procedures in January 2008 and July 2014.

\section{Materials and Methods}

Patients

We retrospectively analysis 193 patients with malignant obstructive jaundice receiving internal-external or external drainage in January 2008 and July 2014. 110 patients with no infection preprocedure were analyzed, 
Table 1. Bacteria Culture Results

\begin{tabular}{lcc}
\hline Bacteriology & $\begin{array}{c}\text { Internal-external } \\
\text { group }\end{array}$ & $\begin{array}{c}\text { External } \\
\text { group }\end{array}$ \\
\hline E. coli & 4 & 3 \\
Dung enterococcus & 5 & 2 \\
Excrement enterococcus & 3 & \\
Giallo enterococcus & 2 & \\
Pseudomonas aeruginosa & 2 & 3 \\
Klebsiella pneumonia coli & 1 & 3 \\
Staphylococcus aureus & 1 & 2 \\
Acinetobacter baumannii & 2 & 2 \\
ESBLs e. coli example & 1 & 1 \\
ESBLs with klebsiella pneumoniae & 1 & 3 \\
\hline
\end{tabular}

including 42 patients with internal-external drainage and 68 patients with external drainage. All patients provided written, informed consent for the procedure, and our institutional review board approved the retrospective review of the patients' medical and imaging records.

\section{Treatment}

PTBD was performed in the interventional diagnosis and treatment center, with the patient under local anesthesia by two interventional radiologist, using continuous fluoroscopy. Intravenous prophylactic antibiotic was given before all procedures using a secondgeneration cephalosporin or according to the oncologist's recommendation. Biliary puncture were carried out using a $21 \mathrm{G}$ Chiba needle through the ninth or tenth intercostal space at the right hemiclavicular line level for the right-lobe bile duct access or by the left anterior subxiphoideal access for left-lobe bile ducts. After bile duct contrastation, a coaxial system (NPAS-100; Cook, Bjaeverskov, Denmark) was introduced and a 0.035-in. angled-tip hydrophilic guidewire (Glidewire; Terumo, Tokyo, Japan) was used to cross the stenotic/obstructive area. During the procedure, if the guide wire could pass through the occlusion and into the duodenum, we prefer to use internal-external drainage, external drainage biliary catheter was placed if the occlusion was not crossed.

\section{Biliary infection diagnostic criteria}

Patients with biliary infection diagnostic criteria after PTBD in 1 month: chills, high fever, with or without abdominal pain, have jaundice, peripheral blood WBC > $10.0 \times 109 / \mathrm{L}$ and neutrophils ratio $>75 \%$, combined with blood and bile bacteria culture results, and exclude other causes of infection.

\section{Bacterial culture and drug susceptibility analysis}

All patients with postprocedure infection were received cultivation of blood and bile bacteriology. Bacterial culture and drug susceptibility analysis: sterile loop line inoculated in sheep blood/rabbit blood AGAR plate within $20 \mathrm{~min}$, and place in the $37^{\circ} \mathrm{C}$ incubation box 24 hours. Choosing appraisal board accord to the colony morphology and using French merry Emmanuel VITEK - AMS60 automatic analyzer to analyze bacteria. Quality control strains were E. coli standard strains (ATCC25922), pseudomonas aeruginosa standard strains (ATCC27853), staphylococcus aureus (ATCC25923). The judgement standard on the basis of the National Committee for Clinical Laboratory Standards (NCCLS). ESBLs test according to NCCLS recommended double paper method, drug sensitive test is Kirby-Bauer diffusion method and drug susceptibility of paper is from oxoid company in America.

\section{Statistical analysis}

Infection incidence parameters were evaluated by t test and $x^{2}$ or Fisher's exact test, and considered as statistically significant if the $\mathrm{P}$ value was under 0.05 . All statistical analyses were done using SPASS 17.0.

\section{Results}

110 patients with malignant obstructive jaundice underwent PTBD were all successful, including 42 patients with internal-external drainage, 68 patients with external drainage, no major complication occured during the process of procedure. According to biliary infection diagnostic criteria after PTBD in one month, germiculture and drug susceptibility analysis. All patients with infection received biliary/blood bacteria culture and drug susceptibility analysis. Experience antibiotics were used before results come out, and sensitive antibiotic treatments were taken until bacteriology results conformed (bacteria culture results are shown in Table 1).

22 patients with infection after procedure in the internal-external drainage group, the infection incidence was $52.4 \%$. 19 patients infection in the external drainage group, the infection incidence was $27.9 \%$. The comparison was statistically significant $(P<0.05) .8$ patients with infection in the internal-external group were under controlled, 14 patients were not controlled (infection control incidence was $36.3 \%$ ). 12 patients with infection in the external group were under controlled, 7 patients were not controlled (infection control incidence was 63.1\%) (P $<0.05$ ). 8 patients (internal-external group was 6 , external group was 2) were dead in one month in 21 patients with infection were not controlled, mainly for septic shock and multiple organ failure. The mortality rate of patients with infection not controlled of internal-external group in one month was $42.8 \%$, while the mortality rate of external group was $28.6 \%$, the comparison was statistically significant $(P<0.05)$.

\section{Discussion}

Malignant obstructive jaundice is a cumbersome complication in patients with advanced solid malignancies. PTBD offers a safe and effective method in providing palliative treatment for patients with biliary obstruction, which can relieve symptoms and restore serum biochemistry to normal. This optimizes the clinical condition of the patients for receiving palliative chemotherapy or radiotherapy, bringing about an improvement in their quality of life (Lofts et al., 1997; Shin et al., 2013). Biliary infection is one of the most common complications after PTBD, also is the important cause leading patients death (Dambraukas et al., 2003). Biliary obstruction could lead cholestasis, while bile is 
a good medium which is advantageous to the bacteria breeding (Herzog et al., 2012). High bilirubin levels is easy to cause bacterial translocation, intestinal flora and damage intestinal mucosa barrier (Ho et al., 2013). Patients' resistance is often poor, which aggravating the possibility of infection.

It is generally believed that there are three possible ways cause infection after PTBD (Ozden et al., 2005; Kaya et al., 2012). The first is bacteria from out of body vitro drainage tube into the bile duct; the second is from intestinal flora retrograde into the bile duct; the third is from blood spread to biliary tract. In our study, we consider that the infection mainly related to retrograde intestinal juice because most of the bacteria come from the intestinal flora according to the result of culture. On the one hand, this may relate to the guide wire enter into the intestinal during procedure and take the intestinal bacteria into the biliary tract when withdraw the wire. On the other hand, intestinal juice may flow into the biliary tract tube when drainage the bile due to the external drainage bag pressure is lower than the pressure in the intestine, which cause infection. And patients with malignant obstructive jaundice generally have weak status, the immune system function is lower, and increases the risk when take a further invasive treatment (Boursier et al., 2009).

It is generally believed that the internal-external drainage conforms to physiological drainage, the patient's infection rates should be lower and get a better prognosis. In previous reports, the incidence of biliary infection after external drainage was $6.5 \%$ - 22\% (Todoroki et al., 2000). Our analysis suggests, however, the infection rate in the internal-external drainage group is $52.4 \%$, significantly higher than the $27.9 \%$ in the external drainage group. The internal-external drainage does not reduce infection incidence after PTBD. Infection is also the independent factor influencing the prognosis of patients with malignant obstructive jaundice (Namias et al., 2005; Zhu et al., 2012). In our study, patients' mortality in the internalexternal group was $42.8 \%$ in one month due to infection was not controlled, significantly higher than the $28.6 \%$ in the external group. The infection rate in the internalexternal group is high, and the infection is not easy to control after procedure, increasing risk of death within one month after the drainage. Other literatures also conform that patients' mortality who receiving internal-external drainage significantly higher than the external drainage or biliary stenting treatment (Simmons et al., 2006; Westwood et al., 2010). The internal-external drainage has no related to be beneficial for the physiological drainage and could achieve good prognosis as we previous thought. We analyze this phenomenon may related to the structure of the internal-external drainage design. Some bile flow into the intestine and also there were some bile flow into to the drainage bag at the same time because we did not shut three-way cock when drainage, this inevitably cause intestinal juice reflux, which greatly increases chances of intestinal retrograde infection, as most of the bacteria from the intestinal which is consistent with the culture results. The infection rate in the external drainage group was lower than those of the internal-external drainage group, because patients with malignant obstructive jaundice received the external drainage, guide wire is not pass the obstructive position into the intestines, and few bacteria come into the biliary tract through wire, reduced the risk of biliary infection. Due to biliary obstruction without opening, also reduce the chances of bacteria retrograde infection. Some scholars suggested that considering the general condition of patients with malignant obstructive jaundice are poor and their survival time are short (Englesbe et al., 2005; Thunyaharn et al., 2013). The external drainage is a good choice to treat the malignant obstructive jaundice, especially combined with patients with preoperative infection, which could significantly reduce the chance of biliary infection caused by bacteria retrograde (Jong et al., 2013).

To sum up, although the internal-external drainage seems to satisfy physiological drainage, the higher infection and mortality rate after drainage affect the longterm prognosis. We believe that patients with malignant obstructive jaundice need to biliary drainage, the external drainage is a good choice, especially combined with patients with preoperative infection, which could significantly reduce the chance of biliary infection caused by bacteria retrograde, increase bile drainage efficiency and improve the long-term prognosis.

\section{References}

Boursier J, Cesbron E, Tropet AL, et al (2009). Comparison and improvement of MELD and Child-Pugh score accuracies for the predietion of 6-month mortality in cirrhotic patients. $J$ Clin Gastroenterol, 43, 580-5.

Dambraukas Z, Paskauskas S, Lizdenis P, et al (2003). Percutaneous transhepatic biliary stenting: the first experience and results of the Hospital of Kaunas University of Medicine. Med, 44, 969-76.

De Jong EA, Moelker A, Leertouwer T, et al (2013). Percutaneous transhepatic biliary drainage in patients with postsurgical bile leakage and nondilated intrahepatic bile ducts. Dig Surg, 30, 444-50.

Englesbe MJ, Dawes LG (2005). Resistant pathogen in biliary obstruction: importance of cultures to guide antibiotic therapy. $H P B, 7,144-8$.

Herzog T, Belyaev O, Hessam S, et al (2012). Bacteribilia with resistant microorganisms after preoperative biliary drainage the influence of bacteria on postoperative outcome. Scand $J$ Gastroenterol, 47, 827-35.

Ho CS, Warkentin AE (2013). Evidence-based decompression in malignant biliary obstruction. Korean J Radiol, 13, S56-61.

Jong Kyoung Choi, Ji Kon Kyu, et al (2013). Palliative treatment of unresectable hepatocellular carcinoma with obstructive jaundice using biliary drainage with subsequent transarterial chemoembolization. J Palliat Med, 16, 1026-33.

Kawakami H, Kondo S, Kuwatani M, et al (2011). Preoperative biliary drainage for hilar cholangiocarcinoma: which stent should be selected? J Hepatobiliary Pancreat Sci, 18, 630-5.

Kaya M, Bestas R, Bacalan F, et al (2012). Microbial profile and antibiotic sensitivity pattern in bile cultures from endoscopic retrograde cholangiography patients. World J Gastroenterol, 18, 3585-9.

Li Sol Y, Kim CW, Jeon UB, et al (2010). Early infectious complications of percutaneous metallic stent insertion for malignant biliary obstruction. AJR Am J Roentgenol, 194, 261-5.

Lofts FJ, Evans TR, Mansi JL, Glees JP, Knight MJ (1997). Bile 


\section{Chuan Xu et al}

duct stents: is there an increased rate of complications in patients receiving chemotherapy? Eur J Cancer, 33, 209-13.

Namias N, Demoya M, Sleeman D, et al (2005). Risk of postoperative infection in patients with bactibilia undergoing surgery for obstructive jaundice. Surg Infect, 6, 323-8.

Ozden I, Tekant Y, Bilge O, et al (2005). Endoscopic and radiologic interventions as the leading causes of severe cholangitis in a tertiary referral center. Am J Surg, 189, 702-6.

Shin Ahn, Yoon-seon Lee, Kyung Soo Lin, et al (2013). Malignant biliary obstructions can we predict immediate postprocedural cholangitis after percutaneous biliary drainage? Support Care Cancer, 21, 2321-6.

Simmons DT, Baron TH, Petersen BT, et al (2006). A novel endoscopic approach to brachytherapy in the management of Hilar cholangiocarcinoma. Am J Gastroenterol, 64, 1792-96.

Thunyaharn N, Promthet S, Wiangnon S, et al (2013). Survival of cholangiocarcinoma patients in northeastern Thailand after supportive treatment. Asian Pac J Cancer Prev, 14, 7029-32.

Todoroki T (2000). Chemotherapy for bile duct carcinoma in the light of adjuvant chemotherapy to surgery. Hepatogastroenterology, 47, 644-9.

Westwood DA, Fernando C, Connor Sj, et al (2010). external percutaneous transhepatic billary drainage for malignant biliary obstruction: a retrospective analysis. J Med Imag Rad Oncol, 54, 108-10.

Xu C, Lv PH, Huang XE, et al (2014). Internal-external percutaneous transhepatic biliary drainage for patients with malignant obstructive jaundice. Asian Pac J Cancer Prev, 15, 9391-4.

Xu C, Lv PH, Huang XE, et al (2014). Analysis of different ways of drainage for obstructive jaundice caused by hilar cholangiocarcinoma. Asian Pac J Cancer Prev , 15, 5617-20.

Xu C, Huang XE, Wang SX, et al (2014). Drainage alone or combined with anti-tumor therapy for treatment of obstructive jaundice caused by recurrence and metastasis after primary tumor resection. Asian Pac J Cancer Prev, 15, 2681-4.

Zhu HD, Guo JH, Teng GJ, et al (2012). A novel biliary loaded 125 I seeds in patients with malignant biliary obstruction: Preliminary results versus a conventional biliary stent. $J$ Hepatol, 56, 1104-11 